\title{
Invariant measures and the compactness of the domain
}

\author{
by Marian Jabłoński (Kraków) and Pawę GóRA (Montreal)
}

\begin{abstract}
We consider piecewise monotonic and expanding transformations $\tau$ of a real interval (not necessarily bounded) into itself with countable number of points of discontinuity of $\tau^{\prime}$ and with some conditions on the variation $V_{[0, x]}\left(1 /\left|\tau^{\prime}\right|\right)$ which need not be a bounded function (although it is bounded on any compact interval). We prove that such transformations have absolutely continuous invariant measures. This result generalizes all previous "bounded variation" existence theorems.
\end{abstract}

1. Introduction. One of the most important problems in ergodic theory is the existence of an invariant measure for a dynamical system.

The topic of this paper is the existence of an absolutely continuous invariant measure for dynamical systems generated by a piecewise expanding transformation of an interval (not necessarily bounded). There are many results in this direction. The first one is Rényi's existence theorem ([Re]) for $\tau:[0,1] \rightarrow[0,1]$ given by

$$
\tau(x)=\phi(x)(\bmod 1),
$$

where $\phi(x):[0,1] \stackrel{\text { onto }}{\longrightarrow}[0, n]$ for some $n \in \mathbb{N}, \phi \in C^{2},\left|\phi^{\prime}\right|>\lambda>1$.

The next significant step was made by Lasota and Yorke [LY] as well as Kosyakin and Sandler [KS], who proved the existence theorem for $\tau$ : $[0,1] \rightarrow[0,1]$ piecewise $C^{2}$ and $\left|\phi^{\prime}\right|>\lambda>1$.

The Lasota-Yorke theorem has many generalizations. One of them was given by Wong [Wo], who assumed that $\tau$ is piecewise $C^{1}$ and $V_{[0,1]}\left(1 /\left|\tau^{\prime}\right|\right)<$ $\infty$. Rychlik [Ry] extended Wong's theorem to a class of transformations with countable partition on a bounded interval. Another generalization of the Lasota-Yorke theorem was given by Keller [Ke] using a kind of generalized variation.

An extension of the Lasota-Yorke theorem in another direction was given by Lasota and Jabłoński [JL] who assumed that $\tau$ is a transformation of $\mathbb{R}$ onto $\mathbb{R}$.

1991 Mathematics Subject Classification: Primary 28D05; Secondary 58F11.

The research of P.G. has been supported by an NSERC grant. 
It seemed that the Lasota-Yorke, Rychlik and Lasota-Jabłoński theorems differ essentially from one another, namely in the countability and finiteness conditions for the corresponding partitions and the compactness conditions for the interval in question, and the questions were:

(a) Is it possible to state in a natural way a theorem which will imply the Lasota-Yorke, Wong, Rychlik and Lasota-Jabłoński theorems?

(b) A very old question: is it possible to weaken the assumptions concerning $\tau$ without loosing the existence of an absolutely continuous invariant measure?

In 1986 Schmitt [Sch] introduced the following global oscillation condition.

Let $\tau: \mathbb{A} \rightarrow \mathbb{A}$ be a transformation of an interval into itself and let $J \subset \mathbb{A}$ be a subinterval; then we define the oscillation of $\tau$ over $J$ as

$$
\operatorname{osc}_{J}\left|\tau^{\prime}\right|=\frac{\max _{J}\left|\tau^{\prime}\right|-\min _{J}\left|\tau^{\prime}\right|}{\min _{J}\left|\tau^{\prime}\right|}
$$

Now, let $\left\{I_{j}\right\}_{j=1}^{\infty}=\mathcal{P}^{(0)}$ be a partition corresponding to $\tau$ such that

a) $I_{j}, j=1,2, \ldots$, are open intervals,

b) $I_{i} \cap I_{j}=\emptyset, i \neq j$,

c) $m\left(\mathbb{A} \backslash \bigcup_{i=1}^{\infty} I_{i}\right)=0$ (the union of $I_{i}$ is almost equal to $\mathbb{A}$ ),

d) $\left.\tau\right|_{I_{i}}$ is of class $C^{1}, i=1,2, \ldots$

The global oscillation of $\tau$ is $\sum_{n=1}^{\infty} d_{n}$, where

and

$$
d_{n}=\sup _{J \in \mathcal{P}^{(n)}} \operatorname{osc}_{J}\left|\tau^{\prime}\right|
$$

$$
\mathcal{P}^{(n)}=\bigvee_{i=0}^{n} \tau^{-i}\left(\mathcal{P}^{(0)}\right)=\left\{\bigcap_{i=0}^{n} \tau^{-i}\left(I_{j(i)}\right): I_{j(i)} \in \mathcal{P}^{(0)}, j_{(i)} \in \mathbb{N}^{n}\right\}
$$

For piecewise $C^{1}$ transformations with bounded global oscillation and finite partition corresponding to $\tau$ Góra [Gó] proved the existence of an absolutely continuous invariant measure. Later Jabłoński, Góra and Boyarsky [JGB] proved the following theorem which is a generalization of the results of Góra's and Jabłoński-Lasota as well as an extension of the result of Rychlik.

Theorem. Let $\tau: \mathbb{A} \rightarrow \mathbb{A}$, where $\mathbb{A}$ is an interval, satisfy the following conditions:

(i) There is a family of intervals $\left\{I_{j}\right\}_{j=1}^{\infty}$ such that
(a) $I_{j}$ is open and $I_{j} \subset A$,
(b) $I_{i} \cap I_{j}=\emptyset$ for $i \neq j$,
(c) $\sup _{i>1} m\left(I_{i}\right)<\infty$,
(d) $m\left(\mathbb{A} \backslash \bigcup_{i=1}^{\infty} I_{i}\right)=0$,

(ii) $\tau_{i}=\left.\tau\right|_{I_{i}}$ is of class $C^{1}$, 
(iii) $\left|\tau_{i}^{\prime}\right| \geq \lambda>2, i \geq 1$,

(iv) the global oscillation is bounded,

(v) $\sup _{i>1}\left|\psi_{1}(x)\right|$ is integrable on $\mathbb{A}$ where $\psi_{i}=\tau_{i}^{-1}$,

(vi) $\sup _{x \in \mathbb{A}} \sup _{i \geq 1}\left|\psi_{i}^{\prime}(x)\right| / m\left(I_{i}\right)=k<\infty$,

(vii)

$$
\sup _{i \geq 1} \int_{|x|>u} \frac{\left|\psi_{i}^{\prime}(x)\right|}{m\left(I_{i}\right)}=k(u) \rightarrow 0 \quad \text { as } u \rightarrow \infty .
$$

Then there is an absolutely continuous $\tau$-invariant finite measure.

The statement of the above theorem is independent of the finiteness of the corresponding partitions and the compactness of $\mathbb{A}$ and implies the Góra and Jabłoński-Lasota theorems as well as an analogue of the Rychlik theorem.

However, there are transformations with unbounded oscillation which have finite absolutely continuous invariant measures. The following example provide us with such a transformation.

EXAMPLE 1. Let $x_{0}$ be such that

$$
\int_{0}^{x_{0}}\left(2+\delta-\frac{1}{\ln t}\right) d t=1
$$

and let

$$
\tau(x)= \begin{cases}\int_{0}^{x}\left(2+\delta-\frac{1}{\ln t}\right) d t & 0 \leq x \leq x_{0}, \\ A\left(x-x_{0}\right)(\bmod 1) & x \in\left[x_{0}, 1\right], A>2,\end{cases}
$$

where $\delta>0$. Then $\tau:[0,1] \rightarrow[0,1], 1 /\left|\tau^{\prime}\right|$ is of bounded variation and the global oscillation is $\approx \sum 1 / n$ and thus unbounded. By the Lasota-Yorke theorem $\tau$ has an absolutely continuous invariant probability measure.

In this paper we prove an analogue of the Jabłoński-Góra-Boyarsky theorem under some assumptions on the variation $V_{[0, x]}\left(1 /\left|\tau^{\prime}\right|\right)$ instead of the boundedness of the oscillation. In particular, these assumptions imply that $V_{I}\left(1 /\left|\tau^{\prime}\right|\right)$ is bounded for any compact interval $I$. In Section 3, modifying $\tau$ from Example 1, we will construct a transformation which satisfies assumptions of Theorem 1 of Section 2 but does not satisfy the assumptions of any other existence theorem. This means that Theorem of [JGB] and the result of this paper are not equivalent and that Theorem 1 is an essential generalization of all previous "bounded variation" existence theorems.

Let us recall here the definition of the space $B V(\mathbb{A})$, which will be used below:

$$
\mathrm{BV}(\mathbb{A})=\left\{f \in L^{1}(\mathbb{A}): \exists g \in L^{1}(\mathbb{A}), g=f \text { a.e., } V_{\mathbb{A}} g<\infty\right\} .
$$


2. Main result. Let $\tau: \bigcup_{i=1}^{\infty} I_{i} \rightarrow \mathbb{A}$ be a transformation satisfying the following conditions:

(1) $I_{i}, i=1,2, \ldots$, are open intervals, $I_{i} \subset \mathbb{A}$,

(2) $\quad I_{i} \cap I_{j}=\emptyset$ for $i \neq j$,

(3) $\sup _{i>1} m\left(I_{i}\right)=L<\infty$, where $m$ is the Lebesgue measure on $\mathbb{R}$,

(4) $\mathbb{A}$ is an interval, not necessarily bounded, and $m\left(\mathbb{A} \backslash \bigcup_{i=1}^{\infty} I_{i}\right)=0$,

(5) $\tau_{i}=\tau_{\mid I_{i}}$ is of class $C^{1}, i=1,2, \ldots$,

(6) $\left|\tau_{i}^{\prime}\right| \geq \lambda>2, i=1,2, \ldots$,

(7) There exist constants $M, \delta, \gamma_{1}, \gamma_{2}, \gamma_{3}$ such that $2 / \lambda+\gamma_{1}+\gamma_{2}+\gamma_{3}<1$ and

(a) for any $i \in J_{1}$, where $J_{1}=\left\{i \in \mathbb{N}: V_{I_{i}}\left(1 /\left|\tau^{\prime}\right|\right) \leq M m\left(I_{i}\right)\right\}$, and for any points $x, x^{\prime} \in I_{i}$ with $\left|x-x^{\prime}\right|<\delta$, we have

$$
V_{\left[x, x^{\prime}\right]} \frac{1}{\left|\tau^{\prime}\right|}<\gamma_{1}
$$

(b) $2 \sum_{i \in J_{2}} V_{I_{i}}\left|\left(1 / \tau^{\prime}\right)\right|<\gamma_{2}$, where $J_{2}=\mathbb{N} \backslash J_{1}$,

(c) $\sum_{i \in J_{3}}\left(\left|\psi_{i}^{\prime}\left(\tau\left(a_{i}\right)\right)\right|+\left|\psi_{i}^{\prime}\left(\tau\left(b_{i}\right)\right)\right|\right)<\gamma_{3}$, where

$$
J_{3}=\left\{i \in \mathbb{N}:\left|\psi_{i}^{\prime}\left(\tau\left(a_{i}\right)\right)\right|>M \cdot m\left(I_{i}\right) \text { or }\left|\psi_{i}^{\prime}\left(\tau\left(b_{i}\right)\right)\right|>M \cdot m\left(I_{i}\right)\right\},
$$
and $\psi_{i}=\tau_{i}^{-1}$.

(8) There exist $W_{1}, W_{2} \subset \mathbb{N}, W_{1} \cap W_{2}=\emptyset, W_{1} \cup W_{2}=\mathbb{N}$, such that the functions $\sup _{k \in W_{1}}\left|\psi_{k}^{\prime}(x)\right| / m\left(I_{k}\right)$ and $\sum_{k \in W_{2}}\left|\psi_{k}^{\prime}(x)\right|$ are integrable.

Theorem 1. Let $\tau: \bigcup_{i=1}^{\infty} I_{i} \rightarrow \mathbb{A}$ be a transformation satisfying conditions (1)-(8). Then there exists a finite absolutely continuous measure $\mu$ on $\mathbb{A}$ invariant with respect to $\tau$.

REMARK. The theorem remains true if an iterate $\tau^{k}$ of $\tau$ satisfies conditions (1)-(8). In particular, it is enough to assume $\lambda>1$ in condition (6) if the iterate $\tau^{k}$ satisfies (7) and (8).

Lemma 1. Let $\tau$ satisfy conditions (1)-(8). Then there exist constants $0<\alpha<1$ and $C>0$ such that

$$
V_{\mathbb{A}} \mathbf{P}_{\tau} f \leq \alpha V_{\mathbb{A}} f+C\|f\|_{1} .
$$

Proof. For $f \in \mathrm{BV}(\mathbb{A})$ the series below are convergent and we have

$$
\begin{aligned}
V_{\mathbb{A}} \mathbf{P}_{\tau} f= & V_{\mathbb{A}} \sum_{i=1}^{\infty} f\left(\psi_{i}(x)\right)\left|\psi_{i}^{\prime}(x)\right| \\
\leq & \sum_{i=1}^{\infty} V_{\tau\left(I_{i}\right)}\left(f \circ \psi_{i}(x)\right)\left|\psi_{i}^{\prime}(x)\right| \\
& +\sum_{i=1}^{\infty}\left(\left|f\left(a_{i}\right)\right| \cdot\left|\psi_{i}^{\prime}\left(\tau\left(a_{i}\right)\right)\right|+\left|f\left(b_{i}\right)\right| \cdot\left|\psi_{i}^{\prime}\left(\tau\left(b_{i}\right)\right)\right|\right)=S_{1}+S_{2},
\end{aligned}
$$


where $I_{i}=\left(a_{i}, b_{i}\right)$. Since $V_{\tau\left(I_{i}\right)}\left|\psi_{i}^{\prime}\right|=V_{I_{i}}\left(1 /\left|\tau^{\prime}\right|\right)<\infty$ we can assume $\psi_{i}^{\prime}\left(\tau\left(a_{i}\right)\right)=0\left(\psi_{i}^{\prime}\left(\tau\left(b_{i}\right)\right)=0\right)$ if $\tau\left(a_{i}\right)= \pm \infty\left(\tau\left(b_{i}\right)= \pm \infty\right)$.

For every $h:[a, b] \rightarrow \mathbb{R}$ with $V_{[a, b]} h<\infty$ there is $c \in[a, b]$ such that

$$
h(c) \leq \frac{1}{m([a, b])} \int_{a}^{b}|h| d m .
$$

For such $c$,

$$
|h(a)| \leq|h(c)|+V_{[a, c]} h \quad \text { and } \quad|h(b)| \leq|h(c)|+V_{[c, b]} h .
$$

Therefore, for each $i \geq 1$ and appropriate $c_{i} \in\left[a_{i}, b_{i}\right]$, we have

$$
\begin{aligned}
& \left|f\left(a_{i}\right)\right| \cdot\left|\psi_{i}^{\prime}\left(\tau\left(a_{i}\right)\right)+\right| f\left(b_{i}\right)|\cdot| \psi_{i}^{\prime}\left(\tau\left(b_{i}\right)\right) \mid \\
& \quad \leq\left(\left|f\left(c_{i}\right)\right|+V_{\left[a_{i}, c_{i}\right]} f\right)\left|\psi_{i}^{\prime}\left(\tau\left(a_{i}\right)\right)\right|+\left(\left|f\left(c_{i}\right)\right|+V_{\left[c_{i}, b_{i}\right]} f\right)\left|\psi_{i}^{\prime}\left(\tau\left(b_{i}\right)\right)\right| \\
& \quad \leq \frac{\left|\psi_{i}^{\prime}\left(\tau\left(a_{i}\right)\right)\right|}{m\left(\left[a_{i}, b_{i}\right]\right)} \int_{a_{i}}^{b_{i}}|f| d m+\frac{\left|\psi_{i}^{\prime}\left(\tau\left(b_{i}\right)\right)\right|}{m\left(\left[a_{i}, b_{i}\right]\right)} \int_{a_{i}}^{b_{i}}|f| d m+\frac{1}{\lambda} V_{\left[a_{i}, b_{i}\right]} f .
\end{aligned}
$$

Let $J_{4}=\mathbb{N} \backslash J_{3}$. By (10) and (7)(c) we obtain

$$
\begin{aligned}
S_{2}= & \left.\sum_{i=1}^{\infty}\left(\left|f\left(a_{i}\right)\right| \cdot\left|\psi_{i}^{\prime}\left(\tau\left(a_{i}\right)\right)\right|+\left|f\left(b_{i}\right)\right| \cdot \mid \psi_{i}^{\prime}\left(b_{i}\right)\right) \mid\right) \\
\leq & \sum_{i \in J_{4}}\left(\frac{\left|\psi_{i}^{\prime}\left(\tau\left(a_{i}\right)\right)\right|}{m\left(\left[a_{i}, b_{i}\right]\right)} \int_{a_{i}}^{b_{i}}|f|+\frac{\left|\psi_{i}^{\prime}\left(\tau\left(b_{i}\right)\right)\right|}{m\left(\left[a_{i}, b_{i}\right]\right)} \int_{a_{i}}^{b_{i}}|f|+\frac{1}{\lambda} V_{I_{i}} f\right) \\
& +\sum_{i \in J_{3}}\left(\sup _{\mathbb{A}}|f|\left(\left|\psi_{i}^{\prime}\left(\tau\left(a_{i}\right)\right)\right|+\left|\psi_{i}^{\prime}\left(\tau\left(b_{i}\right)\right)\right|\right)\right) \\
\leq & 2 M\|f\|_{1}+\frac{1}{\lambda} V_{\mathbb{A}} f+\left(\frac{\|f\|_{1}}{m(\mathbb{A})}+V_{\mathbb{A}} f\right) \cdot \gamma_{3} \\
= & \left(\frac{1}{\lambda}+\gamma_{3}\right) V_{\mathbb{A}} f+\left(2 M+\frac{\gamma_{3}}{m(\mathbb{A})}\right)\|f\|_{1} .
\end{aligned}
$$

We have used the inequality $\sup _{\mathbb{A}}|f| \leq\|f\|_{1} / m(\mathbb{A})+V_{\mathbb{A}} f$ (with $\|f\|_{1} / m(\mathbb{A})$ $=0$ if $m(\mathbb{A})=\infty)$ which holds for functions in $\mathrm{BV}(\mathbb{A})$.

Now we estimate $S_{1}$. Let $\delta$ be as in (7) and $y_{k}=\tau_{i}\left(x_{k}\right)$, where $x_{k} \in I_{i}$.

If $m\left(I_{i}\right) \leq \delta$, we have

$$
\begin{aligned}
V_{\tau\left(I_{i}\right)}\left(f \circ \psi_{i}\right)\left|\psi_{i}^{\prime}\right| & \\
\quad= & \sup _{\tau\left(I_{i}\right)} \sum_{k=1}^{n}\left|\left(f \circ \psi_{i}\right)\left(y_{k}\right)\right| \psi_{i}^{\prime}\left(y_{k}\right)\left|-\left(f \circ \psi_{i}\right)\left(y_{k-1}\right)\right| \psi_{i}^{\prime}\left(y_{k-1}\right)|| \\
& \leq \overline{\sup }_{\tau\left(I_{i}\right)} \sum_{k=1}^{n}\left|\left(f \circ \psi_{i}\right)\left(y_{k}\right)\right| \psi_{i}^{\prime}\left(y_{k}\right)\left|-\left(f \circ \psi_{i}\right)\left(y_{k-1}\right)\right| \psi_{i}^{\prime}\left(y_{k}\right)||
\end{aligned}
$$




$$
\begin{aligned}
& +\overline{\sup }_{\tau\left(I_{i}\right)} \sum_{k=1}^{n}\left|\left(f \circ \psi_{i}\right)\left(y_{k-1}\right)\right| \psi_{i}^{\prime}\left(y_{k}\right)\left|-\left(f \circ \psi_{i}\right)\left(y_{k-1}\right)\right| \psi_{i}^{\prime}\left(y_{k-1}\right)|| \\
\leq & \frac{1}{\lambda} V_{I_{i}} f+\overline{\sup _{I_{i}}} \sum_{k=1}^{n}\left|f\left(x_{k-1}\right)\right|\left|\frac{1}{\left|\tau^{\prime}\left(x_{k}\right)\right|}-\frac{1}{\left|\tau^{\prime}\left(x_{k-1}\right)\right|}\right| \\
\leq & \left.\frac{1}{\lambda} V_{I_{i}} f+\sup _{I_{i}}|f|\right) \overline{\sup _{I_{i}}} \sum_{k=1}^{n}\left|\frac{1}{\left|\tau^{\prime}\left(x_{k}\right)\right|}-\frac{1}{\left|\tau^{\prime}\left(x_{k-1}\right)\right|}\right|,
\end{aligned}
$$

where " $\overline{\sup }_{\tau\left(I_{i}\right)}$ " and " $\overline{\sup }_{I_{i}}$ " indicate the suprema over all finite partitions of $\tau\left(I_{i}\right)$ and $I_{i}$ respectively.

We now consider separately $i \in J_{1}$ and $i \in J_{2}$. If $i \in J_{1}$, then by assumption (7)(a), we can estimate the left hand side of (12) as follows:

$$
\begin{aligned}
V_{\tau\left(I_{i}\right)}\left(f \circ \psi_{i}\right)\left|\psi_{i}^{\prime}\right| & \\
\leq & \frac{1}{\lambda} V_{I_{i}} f+\left(\inf _{I_{i}}|f|+V_{I_{i}} f\right) V_{I_{i}} \frac{1}{\left|\tau^{\prime}\right|} \\
& \leq \frac{1}{\lambda} V_{I_{i}} f+\left(\frac{1}{m\left(I_{i}\right)} \int_{I_{i}}|f|\right) \cdot V_{I_{i}} \frac{1}{\left|\tau^{\prime}\right|}+V_{I_{i}} f \cdot V_{I_{i}} \frac{1}{\left|\tau^{\prime}\right|} \\
& \leq\left(\frac{1}{\lambda}+\gamma_{1}\right) V_{I_{i}} f+M \int_{I_{i}}|f| .
\end{aligned}
$$

If $i \in J_{2}$, then in a standard way, we have

$$
V_{\tau\left(I_{i}\right)}\left(f \circ \psi_{i}\right)\left|\psi_{i}^{\prime}\right| \leq \frac{1}{\lambda} V_{I_{i}} f+\sup _{\mathbb{A}}|f| V_{I_{i}} \frac{1}{\left|\tau^{\prime}\right|} .
$$

If $m\left(I_{i}\right)>\delta$, then there is a partition $a_{i}=c_{0}<c_{1}<\ldots<c_{n_{i}}=b_{i}$ such that

$$
\frac{\delta}{2} \leq\left|c_{j}-c_{j-1}\right|<\delta \quad \text { for } j=1, \ldots, n_{i} .
$$

We have

$$
V_{\tau\left(I_{i}\right)}\left(f \circ \psi_{i}\right)\left|\psi_{i}^{\prime}\right|=\sum_{j=1}^{n_{i}} V_{\tau\left(c_{j-1}, c_{j}\right)}\left(f \circ \psi_{i}\right)\left|\psi_{i}^{\prime}\right| .
$$

In the same way as in (12) we obtain

(16) $V_{\tau\left(c_{j-1}, c_{j}\right)}\left(f \circ \psi_{i}\right)\left|\psi_{i}^{\prime}\right| \leq \frac{1}{\lambda} V_{\left[c_{j-1}, c_{j}\right]} f+\left(\sup _{\left[c_{j-1}, c_{j}\right]}|f|\right) V_{\left[c_{j-1}, c_{j}\right]} \frac{1}{\left|\tau^{\prime}\right|}=R_{i j}$.

Once again we consider separately $i \in J_{1}$ and $i \in J_{2}$. If $i \in J_{1}$, then similarly 
to (13) we have

$$
\begin{aligned}
R_{i j} \leq & \frac{1}{\lambda} V_{\left[c_{j-1}, c_{j}\right]} f+\left(\frac{1}{m\left(\left[c_{j-1}, c_{j}\right]\right)} \int_{c_{j-1}}^{c_{j}}|f|\right) V_{\left[c_{j-1}, c_{j}\right]}\left|\frac{1}{\left|\tau^{\prime}\right|}\right| \\
& +V_{\left[c_{j-1}, c_{j}\right]} f \cdot V_{\left[c_{j-1}, c_{j}\right]} \frac{1}{\left|\tau^{\prime}\right|} \\
\leq & \frac{1}{\lambda} V_{\left[c_{j-1}, c_{j}\right]} f+\frac{2 V_{I_{i}}\left|1 / \tau^{\prime}\right|}{\delta} \int_{c_{j-1}}^{c_{j}}|f|+V_{\left[c_{j-1}, c_{j}\right]} f \cdot \gamma_{1} \\
\leq & \left(\frac{1}{\lambda}+\gamma_{1}\right) V_{\left[c_{j-1}, c_{j}\right]} f+\frac{2 M L}{\delta} \int_{c_{j-1}}^{c_{j}}|f|
\end{aligned}
$$

(the last inequality is a consequence of (15)). Summing up over $j$ 's we get

$$
V_{\tau\left(I_{i}\right)}\left(f \circ \psi_{i}\right)\left|\psi_{i}^{\prime}\right| \leq\left(\frac{1}{\lambda}+\gamma_{1}\right) V_{I_{i}} f+\frac{2 M L}{\delta} \int_{I_{i}}|f| .
$$

If $i \in J_{2}$, then similarly to (14) we have

$$
R_{i j} \leq \frac{1}{\lambda} V_{\left[c_{j-1}, c_{j}\right]} f+\sup _{\mathbb{A}}|f| \cdot V_{\left[c_{j-1}, c_{j}\right]} \frac{1}{\left|\tau^{\prime}\right|}
$$

Summing up over $j$ 's we obtain

$$
V_{\tau\left(I_{i}\right)}\left(f \circ \psi_{i}\right)\left|\psi_{i}^{\prime}\right| \leq \frac{1}{\lambda} V_{I_{i}} f+\sup _{\mathbb{A}}|f| \cdot V_{I_{i}} \frac{1}{\left|\tau^{\prime}\right|} .
$$

Summing up (13) over all $i \in J_{1}$ such that $m\left(I_{i}\right) \leq \delta$, (14) over all $i \in J_{2}$ such that $m\left(I_{i}\right) \leq \delta$, (18) over $i \in J_{1}$ with $m\left(I_{i}\right)>\delta$ and (20) over $i \in J_{2}$ with $m\left(I_{i}\right)>\delta$, and using assumption (7)(b) we obtain

$$
\begin{aligned}
S_{1} & \leq\left(\frac{1}{\lambda}+\gamma_{1}\right) V_{\mathbb{A}} f+\sup _{\mathbb{A}}|f| \cdot \gamma_{2}+\max \left(\frac{2 M L}{\delta}, M\right)\|f\|_{1} \\
& \leq\left(\frac{1}{\lambda}+\gamma_{1}+\gamma_{2}\right) V_{\mathbb{A}} f+\left(\frac{\gamma_{2}}{m(\mathbb{A})}+\max \left(\frac{2 M L}{\delta}, M\right)\right)\|f\|_{1} .
\end{aligned}
$$

Finally, by (11) and (21), we get

$$
\begin{aligned}
V_{\mathbb{A}} \mathbf{P}_{\tau} f \leq & \left(\frac{2}{\lambda}+\gamma_{1}+\gamma_{2}+\gamma_{3}\right) V_{\mathbb{A}} f \\
& +\left(2 M+\frac{\gamma_{3}}{m(\mathbb{A})}+\frac{\gamma_{2}}{m(\mathbb{A})}+\max \left(\frac{2 M L}{\delta}, M\right)\right)\|f\|_{1} .
\end{aligned}
$$

Setting $\alpha=2 / \lambda+\gamma_{1}+\gamma_{2}+\gamma_{3}<1$ and $C=2 M+\gamma_{3} / m(\mathbb{A})+\gamma_{2} / m(\mathbb{A})+$ $\max (2 M L / \delta, M)$ we obtain the assertion of the lemma. 
Lemma 2. If $\tau$ satisfies (1)-(8) and $B \subset L^{1}(\mathbb{A})$ is such that

$$
V_{\mathbb{A}} f+\|f\|_{1} \leq D
$$

for some $D$, then $\mathbf{P}_{\tau} B$ is weakly compact.

Proof. For given $f \in B$ we can choose points $z_{k} \in I_{k}$ such that

$$
\sum_{k} m\left(I_{k}\right)\left|f\left(z_{k}\right)\right| \leq \int_{\mathbb{A}}|f(x)| d x .
$$

We have

$$
\begin{aligned}
\left|\mathbf{P}_{\tau} f\right| & \leq \mathbf{P}_{\tau}|f|=\sum_{k}\left|f\left(\psi_{k}(x)\right)\right| \cdot\left|\psi_{k}^{\prime}(x)\right|=\sum_{k} \frac{\left|\psi_{k}^{\prime}(x)\right|}{m\left(I_{k}\right)} m\left(I_{k}\right)\left|f\left(\psi_{k}(x)\right)\right| \\
& =\sum_{k \in W_{1}} \frac{\left|\psi_{k}^{\prime}(x)\right|}{m\left(I_{k}\right)} m\left(I_{k}\right)\left|f\left(\psi_{k}(x)\right)\right|+\sum_{k \in W_{2}}\left|\psi_{k}^{\prime}(x)\right| \cdot\left|f\left(\psi_{k}(x)\right)\right| \\
& \leq \sup _{k \in W_{1}} \frac{\left|\psi_{k}^{\prime}(x)\right|}{m\left(I_{k}\right)} \sum_{k \in W_{1}} m\left(I_{k}\right)\left|f\left(\psi_{k}(x)\right)\right|+\sup _{\mathbb{A}}|f| \sum_{k \in W_{2}}\left|\psi_{k}^{\prime}(x)\right| .
\end{aligned}
$$

Moreover, by assumption (3),

$$
\begin{aligned}
\sum_{k \in W_{1}} m\left(I_{k}\right)\left|f\left(\psi_{k}(x)\right)\right| & \leq \sum_{k \in W_{1}} m\left(I_{k}\right)\left(\left|f\left(\psi_{k}(x)\right)-f\left(z_{k}\right)\right|+\left|f\left(z_{k}\right)\right|\right) \\
& \leq L V_{\mathbb{A}} f+\|f\|_{1} \leq(L+1) D
\end{aligned}
$$

and

$$
\sup _{\mathbb{A}}|f| \leq \frac{\|f\|_{1}}{m(\mathbb{A})}+V_{\mathbb{A}} f \leq D\left(\frac{1}{m(\mathbb{A})}+1\right) .
$$

Hence, assumption (8) implies uniform integrability of the set $\mathbf{P}_{\tau} B$.

Proof of Theorem 1. For every $f$ of bounded variation with $\|f\|_{1}<\infty$ we have, by Lemma 1 ,

$$
V_{\mathbb{A}} \mathbf{P}_{\tau}^{n} f \leq \alpha^{n} V_{\mathbb{A}} f+\left(\alpha^{n-1}+\alpha^{n-2}+\ldots+1\right) \cdot C \cdot\|f\|_{1} .
$$

Thus for every $n$,

$$
V_{\mathbb{A}} \mathbf{P}_{\tau}^{n} f \leq \frac{C}{1-\alpha}\|f\|_{1},
$$

and $\left\|\mathbf{P}_{\tau}^{n} f\right\|_{1} \leq\|f\|_{1}$.

So, by Lemma 2, the set $\mathbf{P}_{\tau}\left\{\mathbf{P}_{\tau}^{n} f\right\}_{n=0}^{\infty}=\left\{\mathbf{P}_{\tau}^{n} f\right\}_{n=1}^{\infty}$ is weakly compact in $L^{1}(\mathbb{A})$. Since the set of functions of bounded variation is dense in $L^{1}(\mathbb{A})$, using the Kakutani-Yosida Theorem we conclude that for every $f \in L^{1}(\mathbb{A})$ the sequence $\frac{1}{n} \sum_{j=0}^{n-1} \mathbf{P}_{\tau}^{j} f$ converges in $L^{1}(\mathbb{A})$ to a function $Q f \in L^{1}(\mathbb{A})$, and $Q f$ is invariant under $\mathbf{P}_{\tau}$. This completes the proof of Theorem 1 . 
3. Comparison with Jabłoński-Lasota's and Rychlik's theorems. For the convenience of the reader we restate the Jabłoński-Lasota theorem.

Theorem (Jabłoński-Lasota). Let $\left\{I_{i}\right\}_{i=1}^{\infty}$ be a partition of $\mathbb{R}$ and $\tau$ : $\mathbb{R} \rightarrow \mathbb{R}$ be a transformation such that:

(i) $I_{i}$ is an open set for each $i \in \mathbb{N}$;

(ii) $I_{i} \cap I_{j}=\emptyset$ for $j \neq i$;

(iii) $\mathbb{R} \backslash \bigcup I_{i}$ is a countable set;

(iv) $\sup _{i} m\left(I_{i}\right)=L<\infty$ (the partition must actually be infinite);

(v) for any $i, \tau_{i}$ is differentiable and its derivative $\tau_{i}^{\prime}$ is locally Lipschitz;

(vi) $\left|\tau_{i}^{\prime}(x)\right| \geq \lambda>1, x \in \bigcup I_{i}$;

(vii) $\tau_{i}\left(I_{i}\right)=\mathbb{R}$ (a "piecewise onto" condition);

(viii) $\left|\tau_{i}^{\prime \prime}(x)\right| /\left(\tau_{i}^{\prime}(x)\right)^{2} \leq M_{1}<\infty$ (requires the existence of $\tau^{\prime \prime}$ on each $\left.I_{i}\right)$;

(ix) $w(x)=\sup _{i}\left|\psi_{i}^{\prime}(x)\right| / m\left(I_{i}\right)$ is integrable on $\mathbb{R}$.

Then the transformation $\tau$ has a finite absolutely continuous invariant measure.

We will need the following lemma.

Lemma 3. If $\tau$ satisfies conditions (i)-(ix) of the last theorem, then any iteration $\tau^{k}$ of $\tau$ also satisfies these conditions.

Proof. We give the proof for $k=2$. The general case follows by induction.

The transformation $\tau^{2}$ satisfies conditions (i)-(vii) trivially.

It is easy to see that $\left\{I_{i j}=I_{i} \cap \tau_{i}^{-1}\left(I_{j}\right)\right\}_{i, j=1}^{\infty}$ is the partition corresponding to $\tau^{2}$. Since

$$
\begin{aligned}
\frac{\left(\tau^{2}\right)^{\prime \prime}}{\left(\left(\tau^{2}\right)^{\prime}\right)^{2}} & =\frac{\tau^{\prime \prime} \circ \tau \cdot\left(\tau^{\prime}\right)^{2}}{\left(\tau^{\prime} \circ \tau\right)^{2} \cdot\left(\tau^{\prime}\right)^{2}}+\frac{\tau^{\prime} \circ \tau \cdot \tau^{\prime \prime}}{\left(\tau^{\prime} \circ \tau\right)^{2} \cdot\left(\tau^{\prime}\right)^{2}}=\frac{\tau^{\prime \prime} \circ \tau}{\left(\tau^{\prime} \circ \tau\right)^{2}}+\frac{1}{\tau^{\prime} \circ \tau} \cdot \frac{\tau^{\prime \prime}}{\left(\tau^{\prime}\right)^{2}} \\
& \leq M+1 \cdot M=2 M
\end{aligned}
$$

for $x \in I_{i j}, \tau^{2}$ also satisfies (viii). Thus it remains to prove that $\tau^{2}$ satisfies (ix).

First, recall that for any $i \geq 1$ we have

$$
\frac{\sup _{x \in I_{i}}\left|\psi_{i}^{\prime}(x)\right|}{\inf _{x \in I_{i}}\left|\psi_{i}^{\prime}(x)\right|} \leq K
$$

for some constant $K>0$ (see [JGB], Section 3).

The transformation $\tau^{2}$ restricted to $I_{i j}$ is equal to $\tau_{j} \circ \tau_{i}$. Its inverse $\psi_{i j}: \mathbb{R} \rightarrow I_{i j}$ is given by

$$
\psi_{i j}=\left(\tau_{j} \circ \tau_{i}\right)^{-1}=\tau_{i}^{-1} \circ \tau_{j}^{-1}=\psi_{i} \circ \psi_{j} .
$$


We have

$$
m\left(I_{i j}\right)=\int_{I_{j}}\left|\psi_{i}^{\prime}\right| d m=\left|\psi_{i}^{\prime}(\xi)\right| \cdot m\left(I_{j}\right),
$$

for some $\xi \in I_{j}$. Thus, for any $x \in \mathbb{R}$,

$$
\frac{\left|\psi_{i j}^{\prime}(x)\right|}{m\left(I_{i j}\right)}=\frac{\left|\psi_{i}^{\prime}\left(\psi_{j}(x)\right)\right| \cdot\left|\psi_{j}^{\prime}(x)\right|}{\left|\psi_{i}^{\prime}(\xi)\right| \cdot m\left(I_{j}\right)} \leq K \cdot \frac{\left|\psi_{j}^{\prime}(x)\right|}{m\left(I_{j}\right)}
$$

and, consequently,

$$
\sup _{i, j} \frac{\left|\psi_{i j}^{\prime}(x)\right|}{m\left(I_{i j}\right)}
$$

is an integrable function. This completes the proof of the lemma.

Now, we prove that the assumptions of the Jabłoński-Lasota theorem [JL] imply the assumptions of Theorem 1, i.e. that Theorem 1 is a generalization of [JL].

Let $\tau$ satisfy conditions (i)-(ix) of the Jabłoński-Lasota theorem. By Lemma 3, for every $k, \tau^{k}$ also satisfies (i)-(ix) and there is $k$ such that $\left(\tau^{k}\right)^{\prime} \geq \lambda>2$ for $x \in I_{i}^{k}$, where $\left\{I_{i}^{k}\right\}_{i=1}^{\infty}$ is the partition corresponding to $\tau^{k}$. Therefore, in view of the Remark, we can assume that $\tau^{\prime} \geq \lambda>2$.

Now, most of the conditions of Theorem 1 follow trivially from (i)-(ix). We only have to prove condition (7).

Notice that if $\tau^{\prime \prime}$ exists on the intervals $I_{i}, i=1,2, \ldots$, then for any $t, s \in I_{i}$ we have

$$
\left|\frac{1}{\left|\tau^{\prime}(t)\right|}-\frac{1}{\left|\tau^{\prime}(s)\right|}\right|=\frac{\left|\tau^{\prime \prime}(\xi)\right|}{\left(\tau^{\prime}(\xi)\right)^{2}}|t-s|
$$

for some $\xi$ between $t$ and $s$. By assumption (viii), this implies that

$$
V_{\left[x, x^{\prime}\right]} \frac{1}{\left|\tau^{\prime}\right|} \leq M_{1} \cdot\left|x-x^{\prime}\right|,
$$

for any interval $\left[x, x^{\prime}\right] \subset I_{i}, i \geq 1$. By inequality (22), $\tau$ satisfies conditions (7)(a) and (7)(b) with $M=M_{1}, J_{2}=\emptyset, \gamma_{2}=0, \gamma_{1}$ arbitrarily small, and $\delta=\gamma_{1} / M$.

Fix an $i \geq 1$ for a moment. Using (14) again we obtain

$$
V_{I_{i}}\left|\psi_{i}^{\prime}\right| \leq M_{1} \cdot m\left(I_{i}\right)
$$

Hence, since $\tau_{i}\left(I_{i}\right)=\mathbb{R}$ (see (vii)), both the numbers $\left|\psi_{i}^{\prime}\left(\tau\left(a_{i}\right)\right)\right|,\left|\psi_{i}^{\prime}\left(\tau\left(b_{i}\right)\right)\right|$ are equal to 0 . Thus, $J_{3}=\emptyset$ and condition $(7)(\mathrm{c})$ is satisfied trivially.

Now, we discuss the relationship between our result and that of Rychlik $([\mathrm{Ry}])$. Rychlik's result is formulated in a very general way, but in the interval case, the transformation $\tau: \mathbb{A} \rightarrow \mathbb{A}$ is such that $m(\mathbb{A})<\infty$, sup $g<1$ and $V_{\mathbb{A}} g<\infty$, where, $g_{\mid S}=0, g=1 /\left|\tau^{\prime}\right|$ on $\bigcup_{i=1}^{\infty} I_{i}, S=\mathbb{R} \backslash \bigcup_{i=1}^{\infty} I_{i}$ and $\left\{I_{i}\right\}_{i=1}^{\infty}$ is the partition corresponding to $\tau$. 
Similarly to the previous considerations we can assume that Rychlik's transformation satisfies $\left|\tau^{\prime}\right| \geq \lambda>2$ (an analogue of Lemma 3 is contained in Corollary 1 of $[\mathrm{Ry}]$ and can easily be proved directly.) Since conditions (1)-(6) are simple consequences of Rychlik's assumptions we only have to prove (7) and (8).

Rychlik's assumption $V_{\mathbb{A}}\left(1 /\left|\tau^{\prime}\right|\right)<\infty$ implies that

$$
\sum_{i \geq 1} V_{I_{i}} \frac{1}{\left|\tau^{\prime}\right|}<\infty
$$

and

$$
\sum_{i \geq 1}\left(\left|\psi_{i}^{\prime}\left(\tau\left(a_{i}\right)\right)\right|+\left|\psi_{i}^{\prime}\left(\tau\left(b_{i}\right)\right)\right|\right)<\infty .
$$

For arbitrarily small $\gamma_{2}$ and $\gamma_{3}$ we can find $i_{0} \geq 1$ such that the $i_{0}$-tails of the above series are less than $\gamma_{2}$ and $\gamma_{3}$ respectively. For the finite family $\left\{I_{i}\right\}_{i=1}^{i_{0}}$ we can easily find $M, \delta$ and $\gamma_{1}$ such that condition (7) is satisfied.

To prove (8) we use Rychlik's assumption $m(\mathbb{A})<\infty$. Let $W_{2}=\mathbb{N}$. Then we have

$$
\int_{\mathbb{A}} \sum_{k \geq 1}\left|\psi_{k}^{\prime}(x)\right| d m(x) \leq \sum_{k \geq 1} \int_{\mathbb{A}}\left|\psi_{k}^{\prime}(x)\right| d m(x)=\sum_{k \geq 1} m\left(I_{k}\right)=m(\mathbb{A}),
$$

hence the condition (8) is satisfied.

On the other hand, it is not too difficult to show that our conditions imply Rychlik's. So, in fact, for the bounded interval case, both sets of conditions are equivalent.

The following simple example provides us with a transformation which satisfies the assumptions of Theorem 1 but does not satisfy the assumptions of any other theorem mentioned above.

EXAmple 2. Let $\tau: \mathbb{R} \rightarrow \mathbb{R}$ be defined as follows. Let, as in Example 2, $x_{0}$ be such that

$$
\int_{0}^{x_{0}}\left(2+\delta-\frac{1}{\ln t}\right) d t=1
$$

and let

$$
\tau(x)= \begin{cases}\int_{0}^{x}\left(2+\delta-\frac{1}{\ln t}\right) d t, & 0 \leq x \leq x_{0}, \\ A\left(x-x_{0}\right), & x \in\left[x_{0}, 1\right], A>2, \\ \tau(x)=2 \tan \left(\frac{\pi}{2} x\right), & x \in \mathbb{R} \backslash(\mathbb{Z} \cup[0,1]),\end{cases}
$$

where $\delta>0$. 


\section{References}

[Gó] P. Góra, Properties of invariant measures for piecewise expanding transformations with summable oscillation of the derivative, Ergodic Theory Dynam. Systems 14 (1994), 475-492.

[JGB] M. Jabłoński, P. Góra and A. Boyarsky, A general existence theorem for absolutely continuous invariant measures on bounded and unbounded intervals, Nonlinear World 2 (1995), 183-200.

[JL] M. Jabłoński and A. Lasota, Absolutely continuous invariant measures for transformations on the real line, Zeszyty Nauk. Uniw. Jagiell. Prace Mat. 22 (1981), 7-13.

[KS] A. A. Kosyakin and E. A. Sandler, Ergodic properties of a certain class of piecewise smooth transformations of a segment, Izv. Vyssh. Uchebn. Zaved. Mat. 1972, no. 3, 32-40 (in Russian).

[LY] A. Lasota and J. A. Yorke, On the existence of invariant measures for piecewise monotonic transformations, Trans. Amer. Math. Soc. 186 (1973), 481-488.

[Re] A. Rényi, Representations for real numbers and their ergodic properties, Acta Math. Acad. Sci. Hungar. 8 (1957), 477-493.

[Ry] M. R. Rychlik, Bounded variation and invariant measures, Studia Math. 76 (1983), 69-80.

[Sch] B. Schmitt, Contributions à l'étude de systèmes dynamiques unidimensionnels en théorie ergodique, Ph.D. Thesis, University of Bourgogne, 1986.

[Wo] S. Wong, Some metric properties of piecewise monotonic mappings of the unit interval, Trans. Amer. Math. Soc. 246 (1978), 493-500.

Institute of Computer Science

Jagiellonian University

Nawojki 11,

30-072 Kraków, Poland

E-mail: jablonski@softlab.ii.uj.edu.pl
7141 Sherbrooke Street West

Montreal, Quebec

Canada H4B 1R6

E-mail: pgora@vax2.concordia.ca 